\title{
¿RESULTA AFECTADA LA ADAPTACIÓN DE LOS ALUMNOS CUANDO SU CULTURA ES LA MAYORITARIA EN LA ESCUELA?
}

\author{
ARE AFFECTED THE STUDENTS ADJUSTMENT WHEN ITS \\ CULTURE IS THE MAJORITY ONE IN THE SCHOOL?
}

\author{
$M^{a}$ Inmaculada Ramírez*, Francisco Herrera** \\ Universidad de Granada \\ Leandro Navas*** y Juan Luis Castejón**** \\ Universidad de Alicante
}

\section{RESUMEN}

Pretendemos describir los efectos de la agrupación del alumnado en su adaptación, en un contexto educativo multicultural, para diseñar estrategias de intervención educativa. La muestra la forman 1.315 sujetos, mitad cristianos y musulmanes, mitad chicos y chicas, con una edad media de 11 años, escogidos por muestreo estratificado de la población escolar de Ceuta. Con el Inventario de Adaptación de Conducta (De la Cruz y Cordero, 1981) se evalúa la adaptación personal, familiar, escolar, so-

* Ma Inmaculada Ramírez Salguero. Catedrática de E.U. de Psicología Evolutiva y de la Educación, de la Universidad de Granada. Doctora en Filosofía y Ciencias de la Educación por la UNED - Premio Extraordinario-, con líneas de investigación y numerosas publicaciones en el ámbito del aprendizaje, el currículo, la inmigración, la interculturalidad y la convivencia. Miembro numerario del Instituto de Estudios Ceutíes.

** Francisco Herrera Clavero. Catedrático de E.U. de Psicología Evolutiva y de la Educación. Doctor en Filosofía y Ciencias de la Educación por la UNED y Doctor en Psicología por la Universidad de Granada, con líneas de investigación y numerosas publicaciones en el ámbito del aprendizaje, el currículo, la inmigración y la interculturalidad. Miembro de la Junta Rectora del Instituto de Estudios Ceutíes. Vicepresidente de la Asociación INFAD de Psicología.

*** Leandro Navas Martínez. Catedrático de E.U. de Psicología Evolutiva y de la Educación y Secretario de la Facultad de Educación de la Universidad de Alicante. Sus líneas de investigación giran en torno a los determinantes del rendimiento académico, la motivación, la evaluación educativa y la evaluación de programas educativos. Ha dirigido y participado en varios proyectos subvencionados por el CIDE, en proyectos I + D y en investigaciones del Ayuntamiento de Alicante. E-mail: Leandro.Navas@ua.es

**** Juan Luis Castejón Costa. Catedrático de Universidad de Psicología Evolutiva y de la Educación. Sus investigaciones versan sobre rendimiento académico, evaluación de eficacia diferencial entre centros educativos, motivación, aprendizaje y diseño de la instrucción y han sido subvencionadas por entidades como la CICYT, el CIDE, el MCyT y diversas Consejerías de Educación. Actualmente colabora con R. J. Sternberg, de la Universidad de Yale (EE.UU.). 
cial y global. Los resultados muestran que la adaptación de los alumnos cristianos se ve afectada por el agrupamiento, que a los musulmanes no les afecta estar en un contexto mayoritario o no de su propia cultura y que los alumnos cristianos, independientemente de la mayoría dominante, obtienen puntuaciones más elevadas en adaptación personal y familiar, y en los factores contextuales de adaptación siempre que estén en un contexto mayoritario de su cultura.

Palabras clave: Adaptación, agrupación de alumnos, multiculturalidad.

\begin{abstract}
We try to describe the grouping effects of the grouping in the adjustment students, in an multicultural educative context, to design strategies of educative intervention. The sample was composed of 1315 students, half Christian and Muslim, half boys and girls, with an average age of 11 years, that were chosen by stratified sampling of the school population of Ceuta. With the Inventory of Adaptation of Behavior (De la Cruz y Cordero, 1981) the personal, family, school, social and global adaptation was evaluated. The results show that the Christian students adjustment are affected for the cluster, since to the Muslims it doesn't affect them the fact of being contained in a majority context or not of their own culture. And the Christian students always obtain the best results in the personnel and the relative adjustment, and in those contextual factor whenever they are into a majority context of their same culture.
\end{abstract}

Key words: Adjustment, grouping of students, multiculturality.

\title{
Introducción
}

Por diversas razones, nuestros alumnos parecen tener progresivamente mayores dificultades para su adaptación a este mundo tan complejo, donde los cambios se producen a velocidad de vértigo y continuamente. Si esto es así en los centros escolares que pudiéramos calificar como "normales", ¿qué ocurre en los centros donde la pluriculturalidad es la nota singular común, en función de la agrupación de los alumnos y sus proporciones, respecto a la cultura dominante? Este es el problema que aborda este estudio. Es decir, pretendemos averiguar si hay variaciones en las diferentes dimensiones de la adaptación de los alumnos en función de que su cultura sea o no la mayoritaria en la escuela a la que asisten.

En primer lugar, conviene tener en cuenta que la principal función de la educación es la socialización del individuo. Este proceso de integración tiene sus exigencias, el individuo ha de aprenderlas, debe formar los hábitos requeridos y, esos aprendizajes, es la educación quien se los da (Quintana, 1980; García Castaño y Capellán del Toro, 2002; Blanco, 2004; Latorre, 2004). Para ello, la sociedad emplea la iniciación a la vida social por impregnación - la familia, su ambiente y su clima son medios especialmente ricos y complejos de relaciones interpersonales - y por el control de la conducta — adquisición de la capacidad para comportarse de conformidad con las expectativas sociales- (Moraleda, 1987). Está claro el papel decisivo que, en esta adaptación, juega la familia y el estilo educador de los padres (Ramírez, 1997; Balboni y Pedrabissi, 1998; Hickman, Bartholomae y Mckenry, 2000; Roa, Ramírez, Catalá, Campos y Herrera, 2002). Lo que nos cuestionamos es si el grupo cultural dominante en el contexto escolar desempeña, a su vez, un papel igualmente influyente.

En segundo lugar, para analizar el concepto de adaptación-inadaptación se pueden adoptar diferentes criterios. Así, siguiendo un criterio estadístico cuantitativo, "normal" es el comportamiento que reúne las características de la conducta de la mayor parte de un grupo social. Se puede adoptar un criterio estadístico valorativo y, consecuentemente, "normal" es 
todo comportamiento que resulta aprobado por el grupo o que no llega a merecer su rechazo (Sánchez, 1982). También se pueden adoptar enfoques interaccionistas y, entonces, la inadaptación social surge a partir de una situación conflictiva generada de forma permanente entre el individuo y el medio que le rodea (Navarro y Aguilar, 2002). Las relaciones entre personas y situaciones dependen de las condiciones del sistema social en el que están inmersas y la conducta del individuo se convierte en una respuesta adaptativa al ambiente que le circunda. Para Salmerón y Soriano (1989) estas posibles respuestas adaptativas dan lugar a diversos tipos de comportamientos: a) Conformismo previo, caracterizado por la aceptación implícita de la imposibilidad de acceder a determinadas metas culturales, lo que no supone una inadaptación social y rara vez el sujeto entrará en conflicto con las leyes o las instituciones de control de la sociedad; b) Comportamiento antisocial objetivo, manifestado por el sujeto que no acepta la imposibilidad de acceder a las metas sociales y busca caminos alternativos para conseguirlos, y c) Comportamiento social retirado, puesto en práctica por aquellos individuos que, aún sin aceptar el conformismo pasivo, no llegan a desarrollar de forma inevitable un comportamiento antisocial y se alejan de la sociedad.

En tercer lugar, podemos entender la inadaptación como fenómeno que aparece cuando alguien no responde a sus propias expectativas o a las que los demás requieren de él. Si tal situación se da, como individuo (en función de su autoconcepto, de su control emocional, etc.), podríamos hablar de inadaptación personal. Si se produce en su mundo de relaciones, dentro del ámbito familiar, entonces podemos calificarla de inadaptación familiar. Si tiene lugar en el ámbito escolar, la etiquetaríamos de inadaptación escolar. Y, por último, si se manifiesta en el ámbito comunitario, excluidos los dos ámbitos anteriores, suele hablarse de inadaptación social. Queda patente, pues, que cuando el comportamiento que emite el individuo es adecuado a sus propósitos o a las expectativas de los demás, se puede hablar de adaptación. Por tanto, el criterio de inadaptación lo marca la desviación de su comportamiento en relación con las expectativas propias o con las expectativas dominantes en su ambiente. Esas expectativas son extraordinariamente relativas desde un punto de vista cultural y varían con las personas, con el espacio y con el tiempo (Graham y Gisi, 2000; Mathur, 2001; Stoever, 2002; Rice y Slaney, 2002). La variabilidad, a través del tiempo, en las expectativas y en los criterios de adaptación se pone de manifiesto en diferentes estudios (por ejemplo, citando a los más clásicos, Wicman, 1928, o Stouffer, 1952, 1959 y, más recientemente, Ramírez, Herrera y Herrera, 2003).

La valoración cultural, aún siendo relativa, pone en evidencia, también, la existencia de diferentes tipos de inadaptación. Así, por ejemplo, Quay y Quay (1965) determinaron factorialmente tres tipos de inadaptación en el comienzo de la adolescencia. Uno, que podía denominarse inadaptación personal, identificable por aspectos como incapacidad para divertirse, sentimientos de inferioridad, preocupación, timidez, aislamiento, inseguridad, apatía, distracción, etc. Otro, inadaptación social, que abarca aspectos como ser inquieto, reclamar atención, perturbar, hacer ruidos, falta de atención e interés, irresponsabilidad, desobediencia, etc. Y el tercero, que constituye una mezcla de los otros dos.

Del mismo modo, se observan variaciones en las tasas de inadaptación según la clase social, el sexo o la cultura. Al comparar la proporción de escolares inadaptados entre la clase social más alta y la más baja se observa un incremento en esta última cercano al $17 \%$ (Vall, 1973). En cuanto al sexo, los niños muestran un menor control social, mayor agresividad, una actitud menos favorable hacia el aprendizaje y menor satisfacción con el ambiente familiar en comparación con las niñas (Hernández, 1983). Por lo que se refiere a las 
diferencias culturales, dentro del ámbito social, se observa que hay diferencias notables en la valoración del comportamiento por parte de los profesores y por parte de las madres, ya que una misma conducta puede tener distinta tolerancia y diferente significación según los diversos grupos y las distintas clases sociales.

En cuarto lugar, se puede argumentar que el fracaso escolar es una consecuencia de la inadaptación. El niño escolarmente inadaptado presenta síntomas muy variados que van desde las pequeñas indisciplinas en clase al absentismo escolar. El resultado es el retraso académico del niño inadaptado (Ricard, Miller y Heffer, 1995). En el contexto académico, resalta la repercusión de las dificultades de aprendizaje en la adaptación, ya que diversas investigaciones muestran que los niños con dificultades de aprendizaje manifiestan problemas significativos de conducta o déficit en su desarrollo social y son, a menudo, mal aceptados por sus semejantes que no tienen esas dificultades (Bryan, 1974, 1976; Sheare, 1978, Scranton y Ryckman, 1979; Gresham y Reschly, 1986; Sater y Frech, 1989; Bursuck, 1989; Stone y LaGreca, 1990; Bender y Smith, 1990; Vaughn, Hogan, Kouzekanani y Shapiro, 1990; Gresham, 2001; Mateos, 2003). Otros estudios señalan que los niños con dificultades no son menos aceptados, pero sí son más aislados socialmente (Prillaman, 1981). Incluso, se ha comprobado que, aunque los niños con dificultades, como grupo, son menos adaptados socialmente que los niños sin ellas, muchos de ellos no tienen grandes dificultades en las relaciones con sus semejantes (Perlmutter, Crocker, Cordray y Garstecki, 1983; French y Waas, 1985; Kistner y Gatlin, 1989; Sater y French, 1989; Engoron y Sandra, 2001).

A tenor de lo expuesto, la adaptación-inadaptación de un sujeto parece estar sometida a las influencias de la sociedad, de la familia, del ambiente circundante, de la cultura, de las expectativas de los grupos primarios de socialización, de los valores de esos grupos, de la clase social y de variables personales como el género, el fracaso escolar y las dificultades de aprendizaje. Esto nos lleva a plantearnos si la adaptación de los alumnos en contextos pluriculturales varía en función del grupo cultural predominante en la escuela.

En suma, este trabajo persigue tres objetivos. El primero es comprobar si existen diferencias en la adaptación de los escolares pertenecientes a un mismo grupo cultural (comparación intracultural), en función de que, en el centro en el que están escolarizados, la población escolar pertenezca mayoritariamente, o no, a su comunidad de origen. Es decir, dentro de una misma cultura, ¿se producen diferencias entre la adaptación de los estudiantes que asisten a un centro en el que la población escolar que predomina es la propia y la adaptación de los que, por el contrario, acuden a un centro en el que su cultura es minoritaria? El segundo objetivo es verificar si existen diferencias en la adaptación de los alumnos de las distintas comunidades culturales, cuando asisten a un mismo tipo de centro (comparación intercultural) y en éste predomina, o no, la población escolar de una de esas culturas. Es decir, si se compara la adaptación de estudiantes pertenecientes a culturas diferentes que asisten a un mismo tipo de centro ¿se observarán diferencias según la cultura que predomine en el mismo? Y, finalmente, el tercer objetivo es derivar implicaciones educativas en función de las posibles diferencias encontradas.

Para alcanzar estos objetivos se toma, como grupo pluricultural, la población escolar ceutí y, dentro de ella, las culturas cristiana y musulmana. En consecuencia, pretendemos contrastar las hipótesis siguientes:

1. En una sociedad pluricultural, existen diferencias en la adaptación (personal, familiar, escolar, social y global) de los escolares pertenecientes a un mismo grupo cultu- 
ral, en función de que el grupo predominante en el centro educativo al que asisten coincida o no con el suyo. Se presupone que cuando hay coincidencia la adaptación será mayor.

2. En una sociedad pluricultural, existen diferencias en la adaptación (personal, familiar, escolar, social y global) de los escolares de un mismo centro escolar pertenecientes a diferentes grupos culturales, en función de que el grupo predominante en el centro coincida con el suyo o no. Aventuramos que en el grupo en el que hay coincidencia, la adaptación será mayor.

\section{Métodología}

\section{Muestra}

La muestra fue extraída de la población escolar de Ceuta de segundo y tercer ciclo de Educación Primaria y de primer ciclo de E.S.O. $(N=9420)$ por muestreo estratificado con afijación proporcional (según el nivel y la etapa educativa, el grupo cultural — cristiano y musulmán-, y la cultura dominante en el centro educativo); garantizando un nivel de confianza del $95,5 \%$ y un margen de error del 3\%. Del total de los sujetos que componen la muestra $(n=1315)$, el $49,8 \%$ son chicos y el resto chicas; el $50,8 \%$ de ellos pertenecen a la comunidad cristiana y el resto a la musulmana, y están escolarizados en 12 centros educativos diferentes (10 públicos y 2 concertados) en función de la cultura predominante en el mismo, con la siguiente distribución:

a) El 14,6\% procede de un centro en el que prácticamente todos los alumnos son musulmanes (entre el 95 y el 100\%).

b) El 16,1\% asiste a un centro en el que la mayor parte de los alumnos son musulmanes (entre el 75 y el 95\%).

c) El $19,9 \%$ pertenece a un centro en el que predominan los alumnos musulmanes (entre el 50 y el $75 \%$ ).

d) El 19,4\% está escolarizado en un centro en el que predominan los alumnos cristianos (entre el 50 y el 75\%).

e) El 16,1\% está adscrito a un centro en el que la mayor parte de los alumnos son cristianos (entre el 75 y el $95 \%$ ).

f) El 13,8\% procede de dos centros en el que prácticamente todos los alumnos son cristianos (entre el 95 y el 100\%).

\section{Instrumentos y variables}

El instrumento que se emplea es el Inventario de Adaptación de Conducta (IAC), de De la Cruz y Cordero (1981). Está integrado por 123 frases a las que el sujeto debe responder de acuerdo con su manera de pensar y actuar. Su fiabilidad oscila desde 0,81 (para la dimensión de adaptación personal) a 0,97 (para la adaptación global). Con él se evalúan las variables siguientes:

a) Adaptación personal. 
b) Adaptación familiar.

c) Adaptación escolar.

d) Adaptación social.

e) Adaptación global.

En función de la cultura dominante en la escuela a la que asisten los sujetos, se establecen cinco tipos de centros:

1) Centro en el que prácticamente todos los alumnos son musulmanes (entre el 95 y el $100 \%)$.

2) Centro en el que la mayor parte de los alumnos son musulmanes (entre el $75 \mathrm{y}$ el $95 \%)$.

3) Centro en el que predominan los alumnos musulmanes (entre el 50 y el $75 \%$ ).

4) Centro en el que entre el 25 y el 50 por cien de la población escolar es musulmana.

5) Centros en los que entre el 0 y el 25 por cien de la población escolar es musulmana.

\section{Procedimiento y diseño}

Los sujetos responden al IAC en sus aulas habituales, se les garantiza la confidencialidad de sus respuestas y se les demanda la máxima sinceridad. Teniendo en cuenta que no hay una manipulación intencional de las variables y que los grupos no se forman de modo aleatorio, el diseño se ajusta al correlacional básico.

\section{Análisis de datos}

Los datos se someten a análisis diferenciales empleando, como estadístico de contraste de la media, la prueba $t$ de Student para muestras independientes. Se emplea para ello el programa SPSS/PC + (versión 11.5).

\section{Resultados}

Aunque no se han realizado pruebas específicas para comprobar la distribución normal de las variables en la población, tenemos en cuenta el tamaño de la muestra $(n>30)$, y asumimos la normalidad de la distribución, dado que: "una gran mayoría de las características psicológicas tienen una distribución normal en la población" (Seoane, Rechea y Diges, 1982, p. 430). Por ello, los resultados que se ofrecen a continuación provienen de pruebas paramétricas.

En primer lugar, damos cuenta de los resultados obtenidos con los sujetos de la comunidad cristiana, cuando se realizan comparaciones intraculturales para los diversos aspectos de adaptación en función de que el centro al que se asiste sea de mayoría musulmana o no, estableciéndose para ello dos tipos de centros educativos: centros cuya población escolar es mayoritariamente musulmana (entre el 95 y el 100 por ciento de los alumnos pertenecen a 
¿Resulta afactada la adaptación de los alumnos cuando...

esa comunidad) y centros en los que la población escolar musulmana es minoría (entre el 0 y 25 por ciento del total de los alumnos).

Los resultados de la prueba $t$, para el contraste de medias cuando la variable agrupada es la puntuación obtenida en los diferentes factores de adaptación y como variable dicotómica de agrupamiento se considera la cultura dominante en el centro escolar (musulmana o cristiana), se muestran en la tabla 1. La prueba de Levene para la igualdad de varianzas poblaciones no supera el criterio de significación estadística $(p>0,05) \mathrm{y}$, en consecuencia, se asume la igualdad de las mismas.

TABLA 1: Prueba $t$ de Student para el contraste de medias de muestras independientes, considerando como variable de agrupamiento el tipo de centro: Centro donde el alumnado musulmán es mayoría (grupo 1) y centro donde el alumnado musulmán es minoría (grupo 2) y como variable agrupada la puntuación en los diversos factores de adaptación de los alumnos cristianos.

\begin{tabular}{|c|c|c|c|c|c|c|c|}
\hline \multicolumn{2}{|c|}{ Variables } & \multicolumn{3}{|c|}{ Estadísticos } & \multicolumn{3}{|c|}{ Prueba $t$} \\
\hline Adaptación & Grupo & $n$ & Media & $\sigma$ & $t$ & g.l. & $p$ \\
\hline Personal & $\begin{array}{l}1 \\
2\end{array}$ & $\begin{array}{r}14 \\
180\end{array}$ & $\begin{array}{l}42,50 \\
59,34\end{array}$ & $\begin{array}{l}13,26 \\
21,81\end{array}$ & $-4,3$ & 192 & 0,000 \\
\hline Familiar & $\begin{array}{l}1 \\
2\end{array}$ & $\begin{array}{r}14 \\
180\end{array}$ & $\begin{array}{l}44,64 \\
67,34\end{array}$ & $\begin{array}{l}10,08 \\
22,98\end{array}$ & $-7,1$ & 192 & 0,000 \\
\hline Escolar & $\begin{array}{l}1 \\
2\end{array}$ & $\begin{array}{r}14 \\
180\end{array}$ & $\begin{array}{l}36,43 \\
58,61\end{array}$ & $\begin{array}{l}14,47 \\
26,88\end{array}$ & $-5,1$ & 192 & 0,000 \\
\hline Social & $\begin{array}{l}1 \\
2\end{array}$ & $\begin{array}{r}14 \\
180\end{array}$ & $\begin{array}{l}57,07 \\
74,53\end{array}$ & $\begin{array}{l}16,05 \\
20,12\end{array}$ & $-3,2$ & 192 & 0,000 \\
\hline Global & $\begin{array}{l}1 \\
2\end{array}$ & $\begin{array}{r}14 \\
180\end{array}$ & $\begin{array}{l}180,6 \\
259,6\end{array}$ & $\begin{array}{l}37,68 \\
62,67\end{array}$ & $-7,1$ & 192 & 0,000 \\
\hline
\end{tabular}

En ella se observa que los valores de $t$ asociados con las diferencias de medias en adaptación personal, familiar, escolar, social y global son estadísticamente significativos $(p \leq 0,002)$. En todos los factores de adaptación evaluados, los alumnos cristianos que asisten a centros con población escolar mayoritariamente musulmana, obtienen puntuaciones más bajas. Es decir, la adaptación personal, familiar, escolar, social y global de los niños cristianos escolarizados en centros con mayoría musulmana es menor que la de los niños cristianos que acuden a centros en los que su comunidad es mayoritaria y en donde los alumnos musulmanes no predominan.

En segundo lugar, exponemos los resultados obtenidos al realizar comparaciones intraculturales con los sujetos de la comunidad musulmana. En la tabla 2 se resumen los resultados de la prueba $t$ de Student para el contraste de medias cuando la variable agrupada es la puntuación obtenida en cada uno de los factores de adaptación y la variable dicotómica de agrupamiento es el tipo de centro (con mayoría musulmana o sin ella) al que se asiste. La prueba de Levene para la igualdad de las varianzas no supera el criterio de significación estadística $(p>0,05) \mathrm{y}$, por tanto, se asume la igualdad de las varianzas poblacionales. 
TABLA 2: Prueba $t$ de Student para el contraste de medias de muestras independientes, considerando como variable de agrupamiento el tipo de centro: Centro donde el alumnado musulmán es mayoría (grupo 1) y centro donde el alumnado musulmán es minoría (grupo 2) y como variable agrupada la puntuación en los diversos factores de adaptación de los alumnos musulmanes.

\begin{tabular}{|c|c|c|c|c|c|c|c|}
\hline \multicolumn{2}{|c|}{ Variables } & \multicolumn{3}{|c|}{ Estadísticos } & \multicolumn{3}{|c|}{ Prueba $t$} \\
\hline Adaptación & Grupo & $n$ & Media & $\sigma$ & $t$ & g.l. & $p$ \\
\hline Personal & $\begin{array}{l}1 \\
2\end{array}$ & $\begin{array}{r}178 \\
34\end{array}$ & $\begin{array}{l}41,16 \\
43,82\end{array}$ & $\begin{array}{l}23,70 \\
19,15\end{array}$ & $-0,6$ & 210 & 0,538 \\
\hline Familiar & $\begin{array}{l}1 \\
2\end{array}$ & $\begin{array}{r}178 \\
34\end{array}$ & $\begin{array}{l}40,44 \\
37,65\end{array}$ & $\begin{array}{l}21,44 \\
15,24\end{array}$ & $-0,91$ & 210 & 0,366 \\
\hline Escolar & $\begin{array}{l}1 \\
2\end{array}$ & $\begin{array}{r}178 \\
34\end{array}$ & $\begin{array}{l}47,27 \\
48,23\end{array}$ & $\begin{array}{l}18,91 \\
17,96\end{array}$ & $-0,3$ & 210 & 0,784 \\
\hline Social & $\begin{array}{l}1 \\
2\end{array}$ & $\begin{array}{r}178 \\
34\end{array}$ & $\begin{array}{l}75,85 \\
78,82\end{array}$ & $\begin{array}{l}21,33 \\
14,08\end{array}$ & $-1,0$ & 210 & 0,308 \\
\hline Global & $\begin{array}{l}1 \\
2\end{array}$ & $\begin{array}{r}178 \\
34\end{array}$ & $\begin{array}{l}204,6 \\
208,1\end{array}$ & $\begin{array}{l}63,27 \\
45,26\end{array}$ & $-0,4$ & 210 & 0,699 \\
\hline
\end{tabular}

Como se puede observar, los valores de $t$ asociados a las diferencias de las puntuaciones medias en los diferentes factores de adaptación (personal, familiar, escolar, social y global) no son estadísticamente significativos $(p>0,05)$. Parece, por tanto, que, en lo concerniente a las diversas dimensiones de adaptación, no hay diferencias significativas entre los niños musulmanes que asisten a escuelas con mayoría de alumnado musulmán y los niños musulmanes escolarizados en centros en los que esta cultura es minoritaria, por predominar los cristianos entre el alumnado.

Y en tercer lugar, mostramos los resultados de las comparaciones interculturales realizadas en la adaptación de los escolares de las comunidades cristiana y musulmana, según la cultura dominante en el centro al que se asiste (ver tablas 3, 4, 5, 6 y 7). Con los escolares de cada uno de los cinco grupos de colegios, se emplea la prueba $t$ de Student para realizar el contraste de medias para muestras independientes considerando como variable dicotómica de agrupamiento la comunidad de pertenencia (cristiana y musulmana) y como variable agrupada la puntuación alcanzada en cada una de las variables de adaptación consideradas. En todos los casos, la prueba de Levene no resulta estadísticamente significativa y, consecuentemente, se asume que las varianzas poblacionales son iguales.

En la tabla 3 se ofrecen los resultados de la prueba $t$ para el contraste de medias correspondientes a los alumnos de los centros que escolarizan entre un 95 y 100 por cien de alumnos musulmanes.

Como se puede observar en la misma, los valores de $t$ asociados a las diferencias de medias que son estadísticamente significativos son los correspondientes a adaptación escolar $(p=0,037)$, a adaptación social $(p=0,002)$ y a adaptación global $(p=0,045)$. En estos tres factores, la puntuación media de los alumnos cristianos es más baja que la media de los alumnos musulmanes, es decir, están peor adaptados. Parece que cuando el centro acoge a 
TABLA 3: Prueba $t$ de Student para el contraste de medias de muestras independientes, considerando como variable de agrupamiento la cultura de pertenencia: alumnado cristiano (grupo 1) y alumnado musulmán (grupo 2) y como variable agrupada la puntuación en los diversos factores de adaptación de los alumnos. (En el centro están escolarizados entre un 95 y un 100 por cien de alumnos musulmanes).

\begin{tabular}{|c|c|c|c|c|c|c|c|}
\hline \multicolumn{2}{|c|}{ Variables } & \multicolumn{3}{|c|}{ Estadísticos } & \multicolumn{3}{|c|}{ Prueba $t$} \\
\hline Adaptación & Grupo & $n$ & Media & $\sigma$ & $t$ & g.l. & $p$ \\
\hline Personal & $\begin{array}{l}1 \\
2\end{array}$ & $\begin{array}{r}14 \\
178\end{array}$ & $\begin{array}{l}42,50 \\
41,16\end{array}$ & $\begin{array}{l}13,26 \\
23,70\end{array}$ & 0,34 & 190 & 0,739 \\
\hline Familiar & $\begin{array}{l}1 \\
2\end{array}$ & $\begin{array}{r}14 \\
178\end{array}$ & $\begin{array}{l}44,64 \\
40,44\end{array}$ & $\begin{array}{l}10,89 \\
21,44\end{array}$ & 1,34 & 190 & 0,193 \\
\hline Escolar & $\begin{array}{l}1 \\
2\end{array}$ & $\begin{array}{r}14 \\
178\end{array}$ & $\begin{array}{l}36,43 \\
47,27\end{array}$ & $\begin{array}{l}14,47 \\
18,91\end{array}$ & $-2,1$ & 190 & 0,037 \\
\hline Social & $\begin{array}{l}1 \\
2\end{array}$ & $\begin{array}{r}14 \\
178\end{array}$ & $\begin{array}{l}57,07 \\
75,85\end{array}$ & $\begin{array}{l}16,05 \\
21,33\end{array}$ & $-3,2$ & 190 & 0,002 \\
\hline Global & $\begin{array}{l}1 \\
2\end{array}$ & $\begin{array}{r}14 \\
178\end{array}$ & $\begin{array}{l}180,6 \\
204,6\end{array}$ & $\begin{array}{l}37,67 \\
63,27\end{array}$ & $-2,1$ & 190 & 0,045 \\
\hline
\end{tabular}

una amplia mayoría de alumnado musulmán, la comunidad — cristiana o musulmana — a la que pertenece el alumnado introduce diferencias significativas en la adaptación escolar, social y global, puntuando más bajo el alumnado de la comunidad cristiana (que en el centro está en minoría).

En la tabla 4 se resumen los resultados de la prueba $t$ para el contraste de medias, en cada uno de los factores de adaptación, para los alumnos que están escolarizados en centros en los que entre el 75 y el 95 por cien de la población escolar pertenece a la comunidad musulmana.

Se aprecia que los valores de $t$ asociados a las diferencias de medias de adaptación personal, familiar, escolar y global no son estadísticamente significativos $(p>0,05)$. Por el contrario, a la diferencia de medias en adaptación social le corresponden valores de $t$ que son estadísticamente significativos $(p<0,001)$. Observamos que la puntuación media del grupo de alumnos musulmanes es mayor que la media del grupo de los alumnos cristianos, lo que viene a indicar que estos últimos tienen una adaptación social menor.

En la tabla 5 se ofrecen los resultados de la prueba $t$ para el contraste de medias en cada uno de los factores de adaptación, de los niños escolarizados en centros en los que entre el 50 y el 75 por cien de la población escolar pertenecen a la comunidad musulmana.

Como se aprecia en la misma, los valores de $t$ asociados a las diferencias de medias de las puntuaciones de la adaptación escolar y social no superan el criterio de significación estadística $(p=0,181$ y $p=0,962$, respectivamente). Por otra parte, sí superan este criterio los valores de $t$ correspondientes a las diferencias de medias de las puntuaciones de adaptación personal $(p=0,027)$, familiar $(p<0,001)$ y global $(p=0,009)$. En estos tres últimos factores de adaptación mencionados, la puntuación media del grupo de alumnos cristianos es mayor que la puntuación media del grupo de alumnos musulmanes. Puede afirmarse, por 
TABLA 4: Prueba $t$ de Student para el contraste de medias de muestras independientes, considerando como variable de agrupamiento la cultura de pertenencia: alumnado cristiano (grupo 1) y alumnado musulmán (grupo 2) y como variable agrupada la puntuación en los diversos factores de adaptación de los alumnos. (En el centro están escolarizados entre el 75 y el 95 por cien de alumnos musulmanes).

\begin{tabular}{|c|c|c|c|c|c|c|c|}
\hline \multicolumn{2}{|c|}{ Variables } & \multicolumn{3}{|c|}{ Estadísticos } & \multicolumn{3}{|c|}{ Prueba $t$} \\
\hline Adaptación & Grupo & $n$ & Media & $\sigma$ & $t$ & g.l. & $p$ \\
\hline Personal & $\begin{array}{l}1 \\
2\end{array}$ & $\begin{array}{r}32 \\
180\end{array}$ & $\begin{array}{l}37,50 \\
35,58\end{array}$ & $\begin{array}{l}13,85 \\
21,30\end{array}$ & 0,66 & 210 & 0,513 \\
\hline Familiar & $\begin{array}{l}1 \\
2\end{array}$ & $\begin{array}{r}32 \\
180\end{array}$ & $\begin{array}{l}35,28 \\
35,28\end{array}$ & $\begin{array}{l}16,86 \\
22,74\end{array}$ & 0,00 & 210 & 0,999 \\
\hline Escolar & $\begin{array}{l}1 \\
2\end{array}$ & $\begin{array}{r}32 \\
180\end{array}$ & $\begin{array}{l}30,94 \\
36,30\end{array}$ & $\begin{array}{l}13,22 \\
21,77\end{array}$ & $-1,3$ & 210 & 0,179 \\
\hline Social & $\begin{array}{l}1 \\
2\end{array}$ & $\begin{array}{r}32 \\
180\end{array}$ & $\begin{array}{l}48,12 \\
62,50\end{array}$ & $\begin{array}{l}14,52 \\
24,87\end{array}$ & $-4,5$ & 210 & 0,000 \\
\hline Global & $\begin{array}{l}1 \\
2\end{array}$ & $\begin{array}{r}32 \\
180\end{array}$ & $\begin{array}{l}151,8 \\
169,6\end{array}$ & $\begin{array}{l}50,92 \\
76,44\end{array}$ & $-2,7$ & 210 & 0,101 \\
\hline
\end{tabular}

TABLA 5: Prueba $t$ de Student para el contraste de medias de muestras independientes, considerando como variable de agrupamiento la cultura de pertenencia: alumnado cristiano (grupo 1) y alumnado musulmán (grupo 2) y como variable agrupada la puntuación en los diversos factores de adaptación de los alumnos. (En el centro están escolarizados entre el 50 y el 75 por cien de alumnos musulmanes).

\begin{tabular}{|c|c|c|c|c|c|c|c|}
\hline \multicolumn{2}{|c|}{ Variables } & \multicolumn{3}{|c|}{ Estadísticos } & \multicolumn{3}{|c|}{ Prueba $t$} \\
\hline Adaptación & Grupo & $n$ & Media & $\sigma$ & $t$ & g.l. & $p$ \\
\hline Personal & $\begin{array}{l}1 \\
2\end{array}$ & $\begin{array}{r}82 \\
180\end{array}$ & $\begin{array}{l}51,78 \\
44,70\end{array}$ & $\begin{array}{l}23,64 \\
23,99\end{array}$ & 2,23 & 260 & 0,027 \\
\hline Familiar & $\begin{array}{l}1 \\
2\end{array}$ & $\begin{array}{r}82 \\
180\end{array}$ & $\begin{array}{l}55,16 \\
38,87\end{array}$ & $\begin{array}{l}26,42 \\
21,51\end{array}$ & 4,89 & 260 & 0,000 \\
\hline Escolar & $\begin{array}{l}1 \\
2\end{array}$ & $\begin{array}{r}82 \\
180\end{array}$ & $\begin{array}{l}52,07 \\
47,97\end{array}$ & $\begin{array}{l}24,15 \\
22,46\end{array}$ & 1,34 & 260 & 0,181 \\
\hline Social & $\begin{array}{l}1 \\
2\end{array}$ & $\begin{array}{r}82 \\
180\end{array}$ & $\begin{array}{l}70,43 \\
70,58\end{array}$ & $\begin{array}{l}22,58 \\
24,34\end{array}$ & $-0,0$ & 260 & 0,962 \\
\hline Global & $\begin{array}{l}1 \\
2\end{array}$ & $\begin{array}{r}82 \\
180\end{array}$ & $\begin{array}{l}230,1 \\
202,5\end{array}$ & $\begin{array}{l}87,98 \\
73,74\end{array}$ & 2,65 & 260 & 0,009 \\
\hline
\end{tabular}

lo tanto, que la presencia, en mayor o menor medida, de grupos de escolares de la comunidad de procedencia introduce diferencias en las puntuaciones obtenidas por el alumnado en su adaptación (personal, familiar y global). 
¿Resulta afactada la adaptación de los alumnos cuando...

En la tabla 6 mostramos los resultados de la prueba $t$ para el contraste de las puntuaciones medias, en cada uno de los factores de adaptación, cuando los niños están escolarizados en centros en los que entre el 25 y el 50 por cien de la población escolar pertenecen a la comunidad musulmana.

TABLA 6: Prueba $t$ de Student para el contraste de medias de muestras independientes, considerando como variable de agrupamiento la cultura de pertenencia: alumnado cristiano (grupo 1) y alumnado musulmán (grupo 2) y como variable agrupada la puntuación en los diversos factores de adaptación de los alumnos. (En el centro están escolarizados entre el 25 y el 50 por cien de alumnos musulmanes).

\begin{tabular}{|c|c|c|c|c|c|c|c|}
\hline \multicolumn{2}{|c|}{ Variables } & \multicolumn{3}{|c|}{ Estadísticos } & \multicolumn{3}{|c|}{ Prueba $t$} \\
\hline Adaptación & Grupo & $n$ & Media & $\sigma$ & $t$ & g.l. & $p$ \\
\hline Personal & $\begin{array}{l}1 \\
2\end{array}$ & $\begin{array}{r}180 \\
75\end{array}$ & $\begin{array}{l}44,42 \\
31,79\end{array}$ & $\begin{array}{l}20,40 \\
18,30\end{array}$ & 4,64 & 253 & 0,000 \\
\hline Familiar & $\begin{array}{l}1 \\
2\end{array}$ & $\begin{array}{r}180 \\
75\end{array}$ & $\begin{array}{l}52,57 \\
28,71\end{array}$ & $\begin{array}{l}25,22 \\
22,74\end{array}$ & 7,08 & 253 & 0,000 \\
\hline Escolar & $\begin{array}{l}1 \\
2\end{array}$ & $\begin{array}{r}180 \\
75\end{array}$ & $\begin{array}{l}48,94 \\
31,21\end{array}$ & $\begin{array}{l}24,21 \\
22,79\end{array}$ & 5,42 & 253 & 0,000 \\
\hline Social & $\begin{array}{l}1 \\
2\end{array}$ & $\begin{array}{r}180 \\
75\end{array}$ & $\begin{array}{l}74,63 \\
54,83\end{array}$ & $\begin{array}{l}21,92 \\
25,82\end{array}$ & 6,23 & 253 & 0,000 \\
\hline Global & $\begin{array}{l}1 \\
2\end{array}$ & $\begin{array}{r}180 \\
75\end{array}$ & $\begin{array}{l}220,8 \\
146,7\end{array}$ & $\begin{array}{l}71,17 \\
73,69\end{array}$ & 7,51 & 253 & 0,000 \\
\hline
\end{tabular}

Como aparece reflejado en esta tabla, los valores de $t$ correspondientes a las diferencias de medias son estadísticamente significativos en todos los factores evaluados $(p<0,001)$. Las puntuaciones medias en los diversos factores de adaptación considerados son superiores en el grupo de escolares de la comunidad cristiana. Se puede derivar, entonces, que la existencia de grupos de la comunidad de procedencia en la escuela genera diferencias significativas en las puntuaciones de adaptación, mostrando en la misma puntuaciones más altas los alumnos de la comunidad cristiana.

Por último, en la tabla 7 se exponen los resultados de la prueba $t$ para el contraste de medias en cada una de las dimensiones de adaptación evaluadas, referidos a los alumnos que están escolarizados en centros en los que menos del 25 por ciento de la población escolar pertenece a la comunidad musulmana.

Como puede observarse en la misma, los valores de $t$ asociados a las diferencias de medias que resultan significativos son los correspondientes a las dimensiones de adaptación familiar $(p<0,001)$ y social $(p=0,019)$. En el caso de la adaptación familiar la puntuación media es más alta en el grupo de escolares cristianos mientras que, en adaptación social, la puntuación media es más elevada en el grupo de escolares musulmanes. Así, en adaptación familiar y social, que haya en el centro grupos de escolares de la comunidad a la que se pertenece introduce diferencias significativas. 
TABLA 7: Prueba $t$ de Student para el contraste de medias de muestras independientes, considerando como variable de agrupamiento la cultura de pertenencia: alumnado cristiano (grupo 1) y alumnado musulmán (grupo 2) y como variable agrupada la puntuación en los diversos factores de adaptación de los alumnos. (En el centro están escolarizados entre el 0 y el 25 por cien de alumnos musulmanes).

\begin{tabular}{|c|c|c|c|c|c|c|c|}
\hline \multicolumn{2}{|c|}{ Variables } & \multicolumn{3}{|c|}{ Estadísticos } & \multicolumn{3}{|c|}{ Prueba $t$} \\
\hline Adaptación & Grupo & $n$ & Media & $\sigma$ & $t$ & g.l. & $p$ \\
\hline Personal & $\begin{array}{l}1 \\
2\end{array}$ & $\begin{array}{r}180 \\
32\end{array}$ & $\begin{array}{l}47,76 \\
43,59\end{array}$ & $\begin{array}{l}20,90 \\
19,48\end{array}$ & 1,05 & 210 & 0,295 \\
\hline Familiar & $\begin{array}{l}1 \\
2\end{array}$ & $\begin{array}{r}180 \\
32\end{array}$ & $\begin{array}{l}50,39 \\
36,87\end{array}$ & $\begin{array}{l}19,60 \\
15,17\end{array}$ & 3,70 & 210 & 0,000 \\
\hline Escolar & $\begin{array}{l}1 \\
2\end{array}$ & $\begin{array}{r}180 \\
32\end{array}$ & $\begin{array}{l}50,58 \\
48,12\end{array}$ & $\begin{array}{l}21,00 \\
18,48\end{array}$ & 0,62 & 210 & 0,536 \\
\hline Social & $\begin{array}{l}1 \\
2\end{array}$ & $\begin{array}{r}180 \\
32\end{array}$ & $\begin{array}{l}68,24 \\
78,91\end{array}$ & $\begin{array}{l}24,72 \\
14,17\end{array}$ & $-2,4$ & 210 & 0,019 \\
\hline Global & $\begin{array}{l}1 \\
2\end{array}$ & $\begin{array}{r}180 \\
32\end{array}$ & $\begin{array}{l}217,6 \\
207,0\end{array}$ & $\begin{array}{l}64,71 \\
46,21\end{array}$ & 1,12 & 210 & 0,268 \\
\hline
\end{tabular}

\section{Discusión}

Respecto a la primera hipótesis planteada, los resultados obtenidos permiten concluir, en primer lugar, que existen diferencias intraculturales entre los alumnos cristianos escolarizados en centros integrados mayoritariamente por alumnos musulmanes y los que asisten a colegios donde los niños musulmanes son minoría, ya que los primeros obtienen puntuaciones más bajas en su adaptación (en todos los factores - personal, familiar, escolar, social y global-). En segundo lugar, parece que el hecho de que los alumnos musulmanes asistan o no a un centro donde la población escolar sea mayoritariamente musulmana, no parece introducir diferencias intraculturales estadísticamente significativas en su adaptación.

En relación con la segunda hipótesis planteada, los resultados muestran, en primer lugar, que en centros que escolarizan entre el 95 y el 100 por cien de alumnos musulmanes, los alumnos musulmanes obtienen mejores puntuaciones que los alumnos cristianos en su adaptación escolar, social y global. En segundo lugar, en el caso de centros que escolarizan entre el 75 y el 95 por cien de alumnos musulmanes, los alumnos musulmanes obtienen mejores puntuaciones que los alumnos cristianos tan sólo en su adaptación social. En tercer lugar, en los centros que escolarizan entre el 50 y el 75 por cien de alumnos musulmanes, los alumnos cristianos obtienen mejores puntuaciones que los alumnos musulmanes en su adaptación personal, familiar y global. En penúltimo lugar, cuando los centros escolarizan entre el 25 y el 50 por cien de alumnos musulmanes, los alumnos cristianos obtienen mejores puntuaciones que los alumnos musulmanes en todas las facetas de su adaptación. Y por último, en el caso de los centros que escolarizan menos del 25 por cien de alumnos musulmanes, los alumnos cristianos - 
¿Resulta afactada la adaptación de los alumnos cuando...

obtienen significativamente mejores puntuaciones que los alumnos musulmanes en su adaptación familiar y los musulmanes mejores que los cristianos en su adaptación social, lo cual no deja de ser curioso. Probablemente, estos alumnos musulmanes, al ser una minoría muy escasa, se han adaptado a la mayoría social donde se insertan, debido a que, por lo general, son centros que reciben alumnado en mejores condiciones socioeconómicas y educativas.

En vista de ello, en general, parece ser que los datos confirman dos hechos fundamentales:

1. Que los alumnos cristianos son los más afectados en su adaptación en función del agrupamiento, ya que a los musulmanes no les afecta tanto el hecho de estar agrupados de una forma u otra, en un contexto mayoritario o no de su propia cultura.

2. Que los alumnos cristianos, independientemente de la mayoría dominante en la escuela, siempre obtienen los mejores resultados en los factores de adaptación que pudiéramos llamar "primarios" o "próximos", tales como el personal y el familiar, y en los "secundarios" o "contextuales" también, siempre que se hallen insertos o respaldados por un contexto mayoritario de su misma cultura, de lo contrario los alumnos musulmanes destacan, sobre todo, en su adaptación social.

Así pues, dado que la adaptación de todos los alumnos se ve afectada en un sentido o en otro, en función de su agrupamiento, parece congruente sugerir que los estudiantes deberían estar agrupados al 50 por cien en todos los centros educativos pertenecientes a contextos pluriculturales, de manera que las diferentes facetas de la adaptación pudieran quedar tan homogeneizadas como fuera posible; a la vez que se deben diseñar programas de intervención para mejorar la adaptación (en todas sus dimensiones) y para reducir las diferencias entre los alumnos procedentes de comunidades culturales diferentes, ya que parece afectar a la adaptación de los alumnos el hecho de estar en una escuela en la que la mayoría de compañeros pertenecen a una cultura tradicionalmente considerada como minoritaria. En cualquier caso, da la impresión de que la población escolar autóctona es la más vulnerable en su adaptación en contextos pluriculriculturales. No obstante, nuestros resultados deberían ser replicados en otros contextos pluriculturales distintos y considerando otras comunidades culturales de procedencia.

A tenor de estos resultados se pueden aventurar futuras líneas de investigación. ¿Qué ocurriría si desde el centro escolar se trabajara el bilingüismo, de modo que la diversidad lingüística y cultural, en lugar de ser un problema, constituyera un medio para un más completo desarrollo personal, tal y como sugieren López, Serrano, Vila y Castañeda (2004)? Dado que la integración del grupo culturalmente minoritario en la misma escuela a la que asisten los miembros de los grupos de la cultura mayoritaria no parece ser una condición suficiente, aunque sí necesaria, para una buena adaptación, ¿por qué no desarrollar en los centros educativos grupos de trabajo cooperativo de modo que estén interculturalmente constituidos y en los que se llevaran a cabo programas para el desarrollo de habilidades sociales como los propuestos por González y Pelechano (1996), Pelechano y González (1996), Trianes y Muñoz (1994) y Monjas (1993, 1996)? Y, finalmente, poniendo en marcha programas de desarrollo del autoconcepto (Machargo, 1989; Alonso, 2001) ¿se podría mejorar la adaptación de los escolares? Son trabajos que sugerimos con la esperanza de emprenderlos en un futuro no muy lejano. Las sociedades multiculturales, especialmente la ceutí, nos los demandan. 


\section{Referencias bibliográficas}

Alonso, E. (2001). Intervención para la mejora del autoconcepto en alumnos de educación secundaria. Las Palmas de Gran Canaria: Universidad de Las Palmas de Gran Canaria. Servicio de Publicaciones y Producción Documental.

Balboni, G. y Pedrabissi, L. (1998). "School adjustment and academic achievement: parental expectations and socio-cultural background". Early Child Development and Care, 143, 79-93.

Bender, W. N. y Smith, J. K. (1990). "Classroom behavior in children and adolescents with learning disabilities: a meta-analysis". Journal of Learning Disabilities, 23, 235-247.

Blanco, M. (2004). "Integración educativa del inmigrante". En F. Herrera, M. I. Ramírez, J. M. Roa, S. Ramírez y F. Mateos (Coords.), Inmigración, interculturalidad y convivencia III (pp. 55-70). Ceuta: Instituto de Estudios Ceutíes.

Bryan, T. J. (1974). "Peer popularity of learning disabled children". Journal of Learning Disabilities, 7 (10), 621-625.

Bryan, T. J. (1976). "Peer popularity of learning disabled children: A replication". Journal of Learning Disabilities, 9 (5), 307-311.

Bursuck, W. D. (1989). "A comparison of student with learning disabilities to low achieving and higher achieving student on three dimensions of social competence". Journal of Learning Disabilities, 2 (3), 301-318.

De la Cruz, M. V. y Cordero, A. (1981). Inventario de Adaptación de Conducta (IAC). Madrid: T.E.A.

Engoron, M. y Sandra, L. (2001). "Sociocultural adjustment and academic achievement of Mexican males with learning disabilities in U.S. middle schools: Parent and student perspectives". Dissertation Abstracts International: Section A: Humanities and Social Sciences, 61 (10-A), 3858.

French, D. C. y Waas, G. A. (1985). "Teachers ability to identify peer-rejected children: a comparison of sociometrics and teacher rates". Journal of School Psychology, 23 (4), 347-353.

García Castaño, J. y Capellán del Toro, L. (2002). "De la presencia de población inmigrante extranjera en la escuela, al diseño de políticas educativas de igualdad: El caso de Andalucía". En F. Herrera, F. Mateos, S. Ramírez, M. I. Ramírez y J. M. Roa (Coords.), Inmigración, interculturalidad y convivencia I (pp. 81-118). Ceuta: Instituto de Estudios Ceutíes.

González, P. y Pelechano, V. (1996). "Programa Integrado de Entrenamiento en Habilidades Interpersonales en el Ciclo Medio". En V. Pelechano (Dir.). Habilidades Interpersonales. Teoría Mínima y Programas de Intervención. Vol. II. Valencia: Promolibro.

Graham, S. W. y Gisi, S. L. (2000). "The effects of instructional climate and student affairs services on college outcomes and satisfaction". Journal of College Student Development, 41 (3), 279-291.

Gresham, F. M. (2001). "Assessment of social skills in students with emotional and behavioral disorders". Assessment for Effective Intervention, 26 (1), 51-58.

Gresham, F. M., y Reschly, D. J. (1986). "Social skills deficits and low peeracceptance of mainstreamed learning disabled children". Learning Disability Quartely, 9 (2), 156-175.

Hernández, P. (1983). Rendimiento, adaptación e intervención psicoeducativa. Tenerife: Secretariado de Publicaciones de la Universidad de La Laguna.

Hickman, G. P., Bartholomae, S. y Mckenry, P. C. (2000). "Influence of parenting style on the adjustment and academic achievement of traditional college freshmen". Journal of College Student Development, 41 (1), 41-54.

Kistner, J. A. y Gatlin, D. (1989). "Correlates of peer rejection among children with learning disabilities". Learning Disability Quartely, 12 (2), 133-140. 
Latorre, Á. (2004). "Intervención educativa en ámbitos pluriculturales: Pautas prácticas de intervención”. En F. Herrera, M. I. Ramírez, J. M. Roa, S. Ramírez y F. Mateos (Coords.), Inmigración, interculturalidad y convivencia III (pp. 37-54). Ceuta: Instituto de Estudios Ceutíes.

López, M. J., Serrano; M., Vila, I. y Castañeda, E. M. (2004). El bilingüismo. Comunicación presentada en el IV Congreso Nacional sobre Inmigración, Interculturalidad y Convivencia. Ceuta.

Machargo, J. (1989). El profesor y el autoconcepto de sus alumnos. Madrid: Escuela Española.

Mateos, F. (2003). "Las dificultades de aprendizaje en contextos interculturales: El caso de la Ciudad Autónoma de Ceuta". En F. Herrera, F. Mateos, S. Ramírez, M. I. Ramírez y J. M. Roa (Coords.), Inmigración, interculturalidad y convivencia II (pp. 63-94). Ceuta: Instituto de Estudios Ceutíes.

Mathur, S. (2001). "Social and academic school adjustment during early elementary school”. Dissertation Abstracts International: Section B: The Sciences and Engineering, 62 (6-B), 2975.

Mckim, K. S. y Cowen, E. L. (1987). "Multiperspective assesment of young childre'n school adjustment". School Psychology Review, 16 (3), 370-383.

Monjas, M. I. (1993). Programa de enseñanza de habilidades de interacción social. Para niños y niñas en edad escolar. Valladolid: M.I.M.C.

Monjas, M. I. (1996). Programa de enseñanza de habilidades de interacción social (PEHIS) para niños y niñas en edad escolar. Madrid: Ciencias de la Educación Preescolar y Especial.

Moraleda, M. (1987). "Privación cultural, dificultades verbales y fracaso escolar". Bordón, 267, 185198.

Navarro, J. I. y Aguilar, M. (2002). "Conducta antisocial y convivencia”. En F. Herrera, F. Mateos, S. Ramírez, M. I. Ramírez y J. M. Roa (Coords.), Inmigración, interculturalidad y convivencia I (pp. 145-152). Ceuta: Instituto de Estudios Ceutíes.

Pelechano, V. y González, P. (1996). "Programa de entrenamiento en dimensiones de solución de problemas interpersonales". En V. Pelechano (Dir.). Habilidades Interpersonales. Teoría Mínima y Programas de Intervención. Vol. II. Valencia: Promolibro.

Perlmutter, B. F., Crocker, J., Cordray, D. y Garstecki, D. (1983). "Sociometric status and related personality characteristics of mainstreamed learning disabled adolescents". Learning Disability Quartely, 6 (1), 20-30.

Prillaman, D. (1981). "Acceptance of learning disabled student in the mainstream enviroment: A failure to replicate". Journal of Learning Disabilities, 14, 121-132.

Quay, H. C. y Quay, L. C. (1965). "Behavior problems in early adolescence”. Child Development, 36, 368-386.

Quintana, J. M. (1980). Sociología de la educación: La enseñanza como sistema social. Barcelona: Hispano Europea.

Ramírez, M. I. (1997). La adaptación como factor de rendimiento de la población escolar de la comunidad musulmana ceutí. Ceuta: UNED.

Ramírez, M. I., Herrera, F. y Herrera, I. (2003). “¿Qué ocurre con la adaptación y el rendimiento académico de los alumnos, en un contexto educativo pluricultural?". Revista Iberoamericana de Educación de la OEI (on line).

http://www.campus-oei.org/revista/deloslectores.htm\#5

http://www.campus oei.org/revista/edu int2.htm http://www.campus-oei.org/revista/deloslectores/511Ramirez.PDF

Ricard, R. J., Miller, G. A. y Heffer, R.W. (1995). "Developmental trends in the relation between adjustment and academic achievement for elementary school children in mixed-age classrooms". School Psychology Review, 24 (2), 258-270. 
Rice, K. G. y Slaney, R. B. (2002). Clusters of perfectionists: "Two studies of emotional adjustment and academic achievement". Measurement and Evaluation in Counseling and Development, 35 (1), 35-48.

Roa, J. M., Ramírez, M. I., Catalá, A., Campos, C. y Herrera, I. (2002). "Estilo educador de los padres en la sociedad pluricultural ceutí". En F. Herrera, F. Mateos, S. Ramírez, M. I. Ramírez y J. M. Roa (Coords.), Inmigración, interculturalidad y convivencia I (pp. 253-260). Ceuta: Instituto de Estudios Ceutíes.

Salmerón, H. y Soriano, A. (1989). "Diagnóstico de la inadaptación social en el período de la adolescencia". Revista de Educación de la Universidad de Granada, 23 (1), 29-42.

Sánchez, C. (1982). Los problemas del menor inadaptado. Madrid: Consejo Superior de Protección de Menores.

Sater, G. M. y French, D. C. (1989). "A comparison of the social competencies of learning disabled and low achieving elementary children". Journal of Special Education, 23 (1), 29-42.

Scranton, T. R. y Ryckman, D. B. (1979). "Sociometric status of learning disabled children in an integrative program". Journal of Learning Disabilities, 12 (6), 401-407.

Seoane, J., Rechea, C. y Diges, M. (1982). “Conceptos básicos de inferencia estadística”. En J. Seoane (Dir.), Psicología Matemática I (pp. 419-430). Madrid: U.N.E.D.

Sheare, J. B. (1978). "The impact of resource programs upon the self-concept and peer acceptance of learning disabled children". Psychology in the Schools, 15 (3), 406-411.

Stoever, S. A. (2002). "Multiple predictors of college success: Investigation of an empirical model". Dissertation Abstracts International: Section B: The Sciences and Engineering, 62 (7-B), 3367.

Stone, W. L. y Lagreca, A. M. (1990). "The social status of children with learning disabilities: a reexamination". Journal of Learning Disabilities, 23 (1), 32-37.

Stouffer, G. A. W. (1952). "Behavior problems of children as viewed by teachers and mental hygienists". Mental Hygiene, 36, 271-285.

Stouffer, G. A. W. (1959). The attitudes of parents toward certain behavior problems of children, research for the teaching profession. Indiana, Pa: State Teachers College.

Trianes, M. V. y Muñoz, A. (1994). Programa de Educación Social y Afectiva. Málaga: Puerta Nueva, Delegación de Educación y Cultura.

Vall, W. D. (1973). "The problem child schools". London Educational, 2 (2), 3-21.

Vaughn, S., Hogan, F., Kouzekanani, H. y Shapiro, J. (1990). "Peer acceptance, self-perceptions and social skills of learning disabled student prior to identification". Journal of Educational Psychology, 82 (2), 213-221.

Wicman, E. K. (1928). Childrens's behaviour and teacher's attitudes. New York: Commonwealth Fund.

Fecha de recepción: 02-06-03

Fecha de revisión: 14-03-05

Fecha de aceptación: 20-04-05 